\title{
HD 161701, a chemically peculiar binary with a HgMn primary and an Ap secondary $\star, \star \star$
}

\author{
J. F. González ${ }^{1}$, C. Saffe ${ }^{1}$, F. Castelli², S. Hubrig ${ }^{3}$, I. Ilyin $^{3}$, M. Schöller ${ }^{4}$, T. A. Carroll ${ }^{3}$, F. Leone ${ }^{5}$, and M. Giarrusso \\ ${ }^{1}$ Instituto de Ciencias Astronomicas, de la Tierra, y del Espacio (ICATE), 5400 San Juan, Argentina \\ e-mail: fgonzalez@icate-conicet.gob.ar \\ 2 Istituto Nazionale di Astrofisica, Osservatorio Astronomico di Trieste, via Tiepolo 11, 34143 Trieste, Italy \\ 3 Leibniz-Institut für Astrophysik Potsdam (AIP), an der Sternwarte 16, 14482 Potsdam, Germany \\ ${ }_{5}^{4}$ European Southern Observatory, Karl-Schwarzschild-Str. 2, 85748 Garching, Germany \\ 5 Università di Catania, Dipartimento di Fisica e Astronomia, Sezione Astrofisica, via S. Sofia 78, 95123 Catania, Italy \\ ${ }^{6}$ INAF - Osservatorio Astrofisico di Catania, via S. Sofia 78, 95123 Catania, Italy
}

Received 19 July 2013 / Accepted 11 October 2013

\section{ABSTRACT}

\begin{abstract}
Context. In recent years, $\mathrm{HgMn}$ stars have attracted attention after in several of them surface chemical spots of different elements were discovered, whose origin and possible connection with binarity and magnetic fields is not clear yet.

Aims. We perform a complete study of the HgMn binary HD 161701, including the determination of physical parameters and photospheric chemical abundances.

Methods. We analyzed time series of high-resolution spectroscopic observations obtained with ESO's spectrograph FEROS and the spectro-polarimeter HARPS, in addition to a near-IR spectrum obtained with CRIRES. Radial velocity curves for both stars were combined with additional spectroscopic information to estimate absolute stellar parameters. A spectral separation technique was applied, which enabled us to conduct a detailed spectral analysis of both the HgMn primary and the low-luminosity secondary component, which turned out to be a peculiar star as well. Chemical abundances were determined by spectral synthesis. Using HARPS and FEROS spectra obtained at 11 rotational phases, we detected line profile variations in both stellar companions, indicating a nonuniform surface distribution of chemical composition.

Results. HD 161701 is a binary system formed by two chemically peculiar stars in an almost circular orbit with a period of $12.451 \mathrm{~d}$. The primary is a $4.0 M_{\odot}$ star with a chemical pattern typical of HgMn stars with notable overabundances of P, Mn, Ga, Y, Xe, and $\mathrm{Hg}$. The secondary is a $2.4 M_{\odot}$ star showing moderate (1-2 dex) overabundance in metals of the iron group, and strong (2-5 dex) overabundaces in Sr, Y, and particularly rare-earth elements. This star presents a remarkable surface chemical spot pattern with Ti and rare-earths concentrated on the surface region that is pemanently facing the binary companion, and Fe concentrated on the far side. The primary star shows weak profile variations in $\mathrm{Mn}$ and $\mathrm{Cr}$ spectral lines.

Conclusions. The coincidence of chemical spots with the position of the binary companion, a fact that has recently been reported also in a few other chemically peculiar stars, suggests a possible connection between the presence of the companion and the origin of chemical anomalies.
\end{abstract}

Key words. binaries: spectroscopic - stars: chemically peculiar - stars: atmospheres - stars: abundances - stars: magnetic field stars: individual: HD161701

\section{Introduction}

The common assumption when studying the physical properties of stars is that the observed photospheric chemical composition represents their original composition. However, it is well known that a significant fraction of B- and A-type main-sequence stars presents large anomalies in their surface chemical composition, which shows that physical processes occurr that segregate the atomic species throughout the atmosphere. Even though competition between gravitational and radiative forces is generally accepted as the basic explanation of abundance anomalies, the details of these processes are far from being understood. The processes depend on the physical properties and the formation history of the star, giving rise to various groups of chemically

* Based on observations obtained at the European Southern Observatory (ESO programmes 076.D-0172(A), 077.D-0477(A), 089.D-0383(A), and 087-C.0124(A)).

$\star \star$ Tables 3, 6, 7, and plots A and B are only available at the CDS via anonymous ftp to cdsarc.u-strasbg. fr $(130.79 .128 .5)$ or via http://cdsarc.u-strasbg.fr/viz-bin/qcat?]/A+A/561/A63 peculiar stars with distinctive chemical patterns. At the current state of knowledge, the complete characterization of individual peculiar stars, including rotation, multiplicity, age, magnetic fields, and chemical anomalies, is indispensable in order to find empirical correlations that help understanding the role of these properties in generating chemical anomalies.

In early-type chemically peculiar stars, different chemical patterns are observed in some cases in stars of similar ages and masses. Of particular interest is the detection of peculiar stars of different classes in the same stellar system (binaries, multiple stars, open clusters), which can be assumed to have the same original chemical composition and age.

In this paper we present the detailed study of a spectroscopic binary formed by two chemically peculiar components of different type. HD 161701 (=HR 6620) has been classified as a $\mathrm{HgMn}$ binary star. The presence of the $\mathrm{Hg}$ II line at $3984 \AA$ in this star was detected by Cowley et al. (1968), who classified it as $\mathrm{Bp}$ with the presence of $\mathrm{Hg}$ and $\mathrm{Si}$, and later as $\mathrm{B} 9 \mathrm{p} \mathrm{HgMn}$ (Cowley et al. 1969). Shortly after this, HD 161701 was reported as a single-lined spectroscopic binary by Hube (1969), 


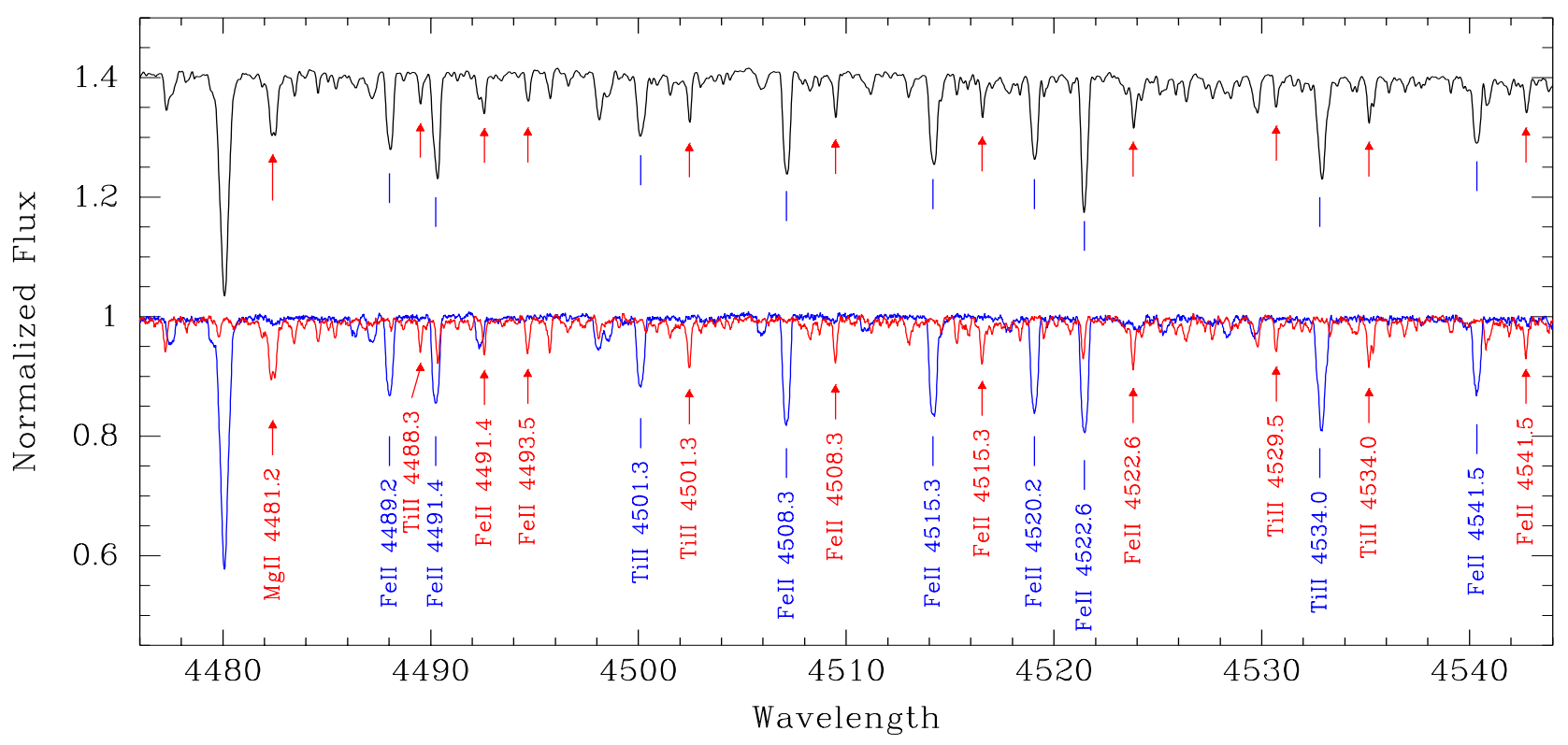

Fig. 1. HARPS spectrum of HD 161701 taken near quadrature at $\phi 0.215$ (in black, shifted vertically by 0.4 ). Several spectral lines of the primary and secondary components are marked with blue ticks and red arrows, respectively. For a better visualization of the composite spectrum, the reconstructed spectra of the primary (blue) and secondary (red) stars are overplotted in the lower part.

who calculated an orbit with a period of 12.452 days and a semiamplitude of about $53 \mathrm{~km} \mathrm{~s}^{-1}$ based on radial velocities (RVs) measured in 42 plates. Aikman (1976) recalculated the orbit, obtaining $P=12.4515 \pm 0.0008 \mathrm{~d}$ and $K=59.7 \pm 0.6 \mathrm{~km} \mathrm{~s}^{-1}$. Since then, no other orbital study of this star has been carried out; in particular, there are no velocity measurements for the secondary star. The chemical anomalies have not been studied in detail, but they are registered in the Renson \& Manfroid (2009) catalog as B9 $\mathrm{Hg} \mathrm{Mn} \mathrm{Si,} \mathrm{according} \mathrm{to} \mathrm{the} \mathrm{works} \mathrm{by} \mathrm{Cowley} \mathrm{et} \mathrm{al.}$

In this study we perform a detailed analysis of both stellar components using high-resolution spectroscopic observations in optical and infrared wavelengths (Sects. 2 and 4, respectively). We calculate orbital parameters (Sect. 3) and estimate physical parameters for both stars (Sect. 6). A chemical abundance analysis is performed for both stellar components (Sect. 7), revealing that the secondary star is a chemically peculiar star as well. In both stars, surface chemical inhomogeneities are detected through the variation of spectral line profiles (Sect. 5). The results on the measurements of the magnetic fields of the two components using HARPS spectropolarimetric observations will be reported in a separate article.

Recent results (e.g. Hubrig et al. 2011; Nuñez et al. 2011) show that line profile variability is a typical characteristic of HgMn stars, and not an exception. This variability is caused by an inhomogeneous chemical-element distribution and implies that most $\mathrm{HgMn}$ stars present a nonuniform distribution of one or more chemical elements. To our knowledge, HD 161701 is the first spectroscopic binary with a $\mathrm{HgMn}$ primary and a classical Ap star as a secondary component. Among the currently studied companions in SB2 systems, none showed chemical peculiarities typical for classical Ap stars with a large overabundance of rare-earth elements. Thus, the study of the system HD 161701 allows us for the first time to learn about the effect of binarity on the development of surface chemical inhomogeneities on the surface of companions of different type of peculiarity.

\section{Observations}

As part of our survey of spectral variability in HgMn stars, HD 161701 was observed with the echelle spectrograph FEROS at the $2.2 \mathrm{~m}$ telescope at La Silla. Six spectra were taken in two runs in October 2005 and September 2006. Their wavelength range is $3530-9220 \AA$, and the nominal resolving power is 48000 . These data were reduced with standard IRAF tasks. The inspection of the spectral behaviour of both components in HD 161701 revealed not only strong variability of the line profiles in the secondary component, but also a strong overabundance of rare-earth elements, such as $\mathrm{Eu}, \mathrm{Nd}$, Pr, and Sm.

Motivated by the discovery of the outstanding peculiar nature of the secondary component, we obtained in 2012 six high-resolution spectra taken on consecutive nights with the HARPSpol polarimeter (Snik et al. 2011) that feeds the HARPS spectrometer (Mayor et al. 2003) at the ESO 3.6-m telescope in La Silla. These spectra have a resolving power of $R=110000$ and cover the wavelength range $3780-6913 \AA$ with a small gap around $5300 \AA$. The reduction was performed using the HARPS data reduction software available at the ESO 3.6-m telescope in La Silla.

Additionally, near-infrared observations in a few spectral regions were obtained with the spectrograph CRIRES at the ANTU telescope on Cerro Paranal, using a slit width of $0.2^{\prime \prime}$. The achieved resolution is slightly above 90000 , determined from weak telluric lines in the reduced CRIRES spectra. Routines of the ESO CRIRES pipeline software were used for the data reduction.

\section{Radial velocities and orbital analysis}

The spectral lines of the secondary component, not detected in previous studies, are clearly visible in our spectra. As an illustration, Fig. 1 shows a small spectral region where the several spectral lines of Fe II, Ti II, and Mg II for the primary and the secondary linesets are clearly distinguished.

To appropriately measure the RVs and separate the spectra of the two components, we applied the technique described in González \& Levato (2006) separately to the HARPS and FEROS datasets. For the cross-correlations used by this method in the RV calculation, standard templates taken from the Pollux database were used (Palacios et al. 2010). In measuring RVs 
Table 1. Spectroscopic observations and radial velocities.

\begin{tabular}{|c|c|c|c|c|c|c|c|c|c|c|}
\hline MJD & Phase & $\begin{array}{c}\mathrm{RV}_{\mathrm{A}} \\
\mathrm{km} \mathrm{s}^{-1}\end{array}$ & $\begin{array}{c}\mathrm{err}_{\mathrm{A}} \\
\mathrm{km} \mathrm{s}^{-1}\end{array}$ & $\begin{array}{l}\mathrm{RV}_{\mathrm{B}} \\
\mathrm{km} \mathrm{s}^{-1}\end{array}$ & $\begin{array}{c}\mathrm{err}_{\mathrm{B}} \\
\mathrm{km} \mathrm{s}^{-1}\end{array}$ & $S / N_{440}$ & $S / N_{600}$ & $\begin{array}{r}(\mathrm{O}-\mathrm{C})_{\mathrm{A}} \\
\mathrm{km} \mathrm{s}^{-1}\end{array}$ & $\begin{array}{r}(\mathrm{O}-\mathrm{C})_{\mathrm{B}} \\
\mathrm{km} \mathrm{s}^{-1}\end{array}$ & Instr. \\
\hline 53664.0153 & 0.7050 & 38.76 & 0.11 & -116.09 & 0.13 & 180 & 325 & -0.08 & -0.31 & FEROS \\
\hline 53665.0133 & 7851 & 39.70 & 0.09 & -117.85 & 0.15 & 215 & 340 & -0.19 & -0.09 & FEROS \\
\hline 53704.3050 & 0.9408 & 3.2 & 0.9 & -60.9 & 0.7 & $450^{a}$ & $520^{a}$ & 0.2 & -4.9 & CRIRES \\
\hline 53979.0805 & 0.0089 & -21.58 & 0.09 & -13.99 & 0.16 & 295 & 395 & 0.25 & 0.80 & FEROS \\
\hline 53986.0706 & 0.5703 & 6.50 & 0.09 & -61.73 & 0.17 & 125 & 240 & -0.04 & -0.15 & FEROS \\
\hline 53986.0818 & 0.5712 & 6.86 & 0.09 & -62.22 & 0.17 & 125 & 240 & 0.01 & -0.12 & FEROS \\
\hline 53987.0870 & 0.6519 & 29.97 & 0.09 & -101.54 & 0.16 & 155 & 275 & -0.10 & -0.09 & FEROS \\
\hline 56143.2012 & 0.8167 & 36.34 & 0.09 & -111.59 & 0.14 & 150 & 210 & 0.08 & 0.08 & HARPS \\
\hline 56144.0997 & 0.8888 & 20.33 & 0.09 & -84.35 & 0.16 & 145 & 220 & 0.16 & 0.21 & HARPS \\
\hline 56145.0725 & 0.9670 & -5.58 & 0.10 & -40.27 & 0.18 & 125 & 185 & 0.38 & 0.30 & HARPS \\
\hline 56146.1198 & 0.0511 & -37.57 & 0.11 & 12.93 & 0.19 & 125 & 195 & -0.32 & 0.80 & HARPS \\
\hline 56147.1442 & 0.1334 & -63.00 & 0.11 & 55.35 & 0.17 & 130 & 210 & 0.08 & -0.27 & HARPS \\
\hline 56148.1297 & 0.2125 & -77.21 & 0.12 & 79.07 & 0.14 & 140 & 265 & -0.07 & -0.22 & HARPS \\
\hline
\end{tabular}

Notes. ${ }^{(a)}$ At $\lambda=1090 \mathrm{~nm}$ and $1570 \mathrm{~nm}$, respectively.

Table 2. Orbital parameters.

\begin{tabular}{lc}
\hline \hline$P[\mathrm{~d}]$ & $12.45123 \pm 0.00004$ \\
$T_{\mathrm{I}}$ & $53891.811 \pm 0.0012$ \\
$V_{\mathrm{o}}\left[\mathrm{km} \mathrm{s}^{-1}\right]$ & $-19.00 \pm 0.12$ \\
$K_{\mathrm{A}}\left[\mathrm{km} \mathrm{s}^{-1}\right]$ & $60.24 \pm 0.29$ \\
$K_{\mathrm{B}}\left[\mathrm{km} \mathrm{s}^{-1}\right]$ & $101.31 \pm 0.26$ \\
$e$ & $0.0043 \pm 0.0023$ \\
$\omega[\mathrm{rad}]$ & $5.39 \pm 0.45$ \\
$a \sin i\left[R_{\odot}\right]$ & $39.74 \pm 0.10$ \\
$M_{\mathrm{A}} \sin ^{3} i\left[M_{\odot}\right]$ & $3.411 \pm 0.023$ \\
$M_{\mathrm{B}} \sin ^{3} i\left[M_{\odot}\right]$ & $2.028 \pm 0.018$ \\
$q$ & $0.595 \pm 0.003$ \\
\hline
\end{tabular}

we decided to use templates with solar abundances, instead of synthetic spectra calculated specifically for the observed abundance pattern, with the intention of minimizing the influence of chemical spots, which are more notable in elements with peculiar abundances.

Since a systematic RV difference of a few tenths of $\mathrm{km} \mathrm{s}^{-1}$ has been found between FEROS and HARPS spectra, we applied a general correction to each dataset using the RVs of telluric lines. These corrections were determined with an accuracy of 14 and $22 \mathrm{~m} \mathrm{~s}^{-1}$ for HARPS and FEROS data, respectively. Interstellar Na I lines gave an additional verification for the consistence of the RV zero. Table 1 lists the obtained RVs and their formal errors.

A Keplerian orbit was fitted to our RV data with the leastsquares method, obtaining the following parameters, whose values are listed in Table 2: $P$ (period), $T_{\mathrm{I}}$ (MJD for the primary conjunction), $V_{\mathrm{o}}$ (center-of-mass velocity), $K_{\mathrm{A}}$ and $K_{\mathrm{B}}$ (amplitudes of RV curves), $e$ (orbital eccentricity), $\omega$ (argument of periastron), and $q$ (mass-ratio).

The RV curves are shown in Fig. 2. The previous observations by Hube (1969) and Aikman (1976) are fully consistent with our orbit, indicating that there is no significant long-term variability effect on the orbit. The RMS of the RV residuals are 0.19 and $0.39 \mathrm{~km} \mathrm{~s}^{-1}$ for the primary and secondary star, respectively. The eccentricity is only marginally different from zero, but, as discussed in Sect. 5.3, we consider that the orbit has probably not been fully circularized yet.

\section{Infrared spectroscopy}

The IR observations were obtained with the near-infrared spectrograph CRIRES using a slit width of 0.2 arcsec. Routines of

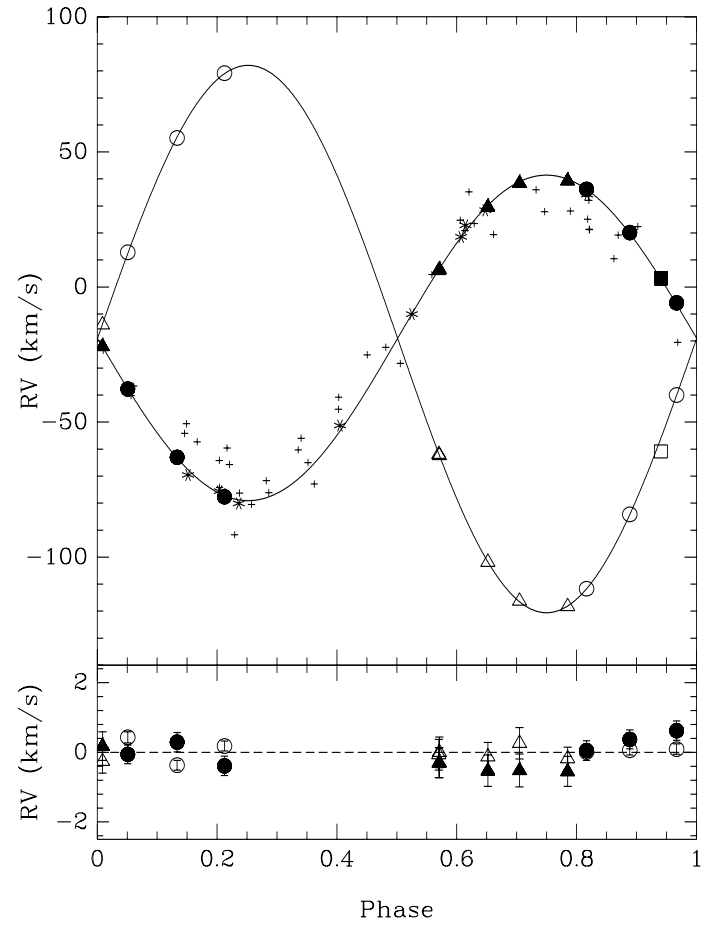

Fig. 2. Radial velocity curves. Triangles and circles are FEROS and HARPS observations, respectively. Filled (open) symbols correspond to the primary (secondary) component. The primary RV measured in the CRIRES spectrum is marked with a filled square. Small crosses are RVs from Hube (1969) and asterisks from Aikman (1976). Residuals (observed - calculated) are shown for our HARPS and FEROS observations in the lower panel.

the ESO CRIRES pipeline software were used for data reduction. The final spectrum covers approximatelly the spectral windows $1065-1108 \mathrm{~nm}, 1550-1587 \mathrm{~nm}$, and 2276-2325 nm with a resolving power of about 95000 .

In spectral regions with several telluric lines, the wavelength scale was recalibrated using telluric lines. We used low-order (2-3) polynomials and typically obtained residuals of about $0.0005-0.0020 \mathrm{~nm}$. Telluric features were removed using hot fast-rotating standard stars observed at similar zenith distances. After moving the wavelength scale to the topocentric frame of rest, we averaged the normalized spectra of several standard stars to calculate a master spectrum of telluric lines. To match the 

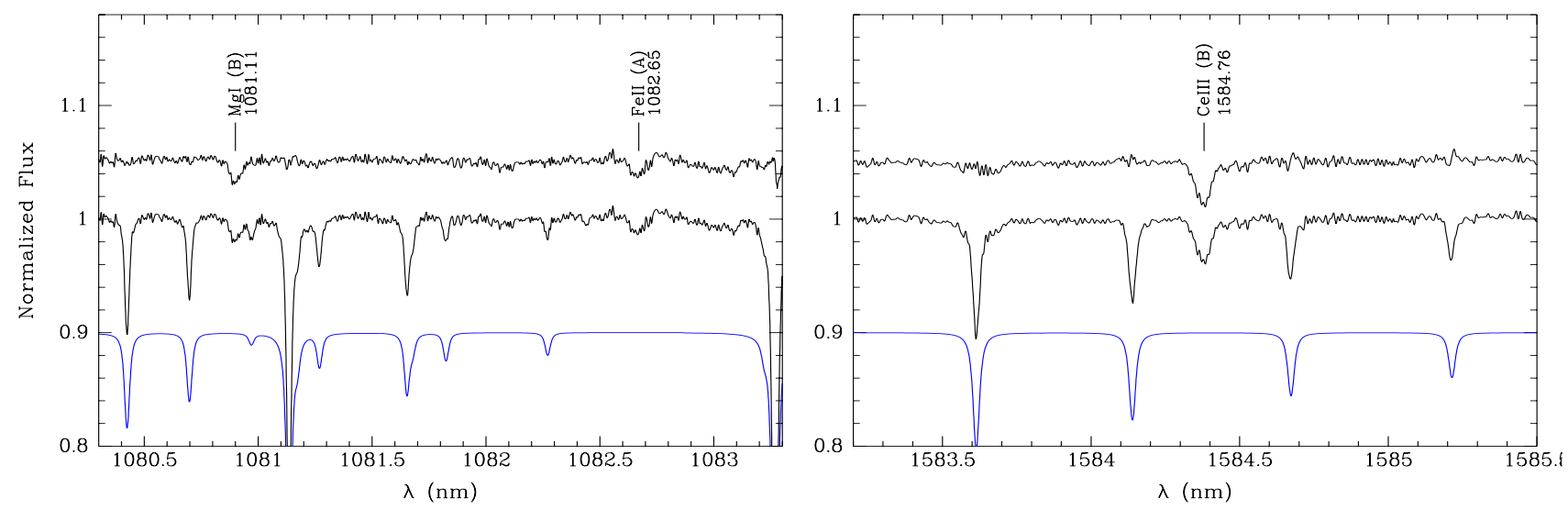

Fig. 3. Sections of the CRIRES spectrum showing some of the identified lines in components A and B. From bottom to top: telluric spectrum, observed spectrum, and cleaned spectrum.

telluric line intensity of each object spectrum, this master telluric spectrum was scaled by increasing it to an appropriate power. Then, each object spectrum was divided by its telluric spectrum to obtain a spectrum essentially free of telluric lines. In this way, the corrected object spectrum $S_{\text {corr }}$ was calculated from the observed spectrum $S_{\text {obs }}$ and the telluric line master spectrum $T$ as $S_{\text {corr }}=S_{\text {obs }} \cdot T^{-\alpha}$,

where $\alpha$ is the ratio of optical thickness of the terrestrial atmosphere in the spectra $S_{\text {obs }}$ and $T$, respectively. The coefficient $\alpha$ was chosen trying to obtain a flat spectrum in the regions without stellar lines.

We identified stellar lines using the spectrum synthesis method. Lines of $\mathrm{He}, \mathrm{C}, \mathrm{Mg}, \mathrm{Si}, \mathrm{Fe}$, and $\mathrm{Sr}$ were identified in the primary component, while $\mathrm{C}, \mathrm{O}, \mathrm{Mg}, \mathrm{Si}, \mathrm{Ti}, \mathrm{Sr}$, and $\mathrm{Ce}$ are visible in the secondary star. All identified lines are listed in Table 3. Figure 3 shows two spectral regions containing some of the identified lines.

We derived the RV of both stellar companions in our infrared spectrum by measuring individual spectral lines. We obtained for the primary $3.2 \pm 0.9 \mathrm{~km} \mathrm{~s}^{-1}$ from $3 \mathrm{Fe}$ and $\mathrm{H}$ lines and $-60.9 \pm 0.7 \mathrm{~km} \mathrm{~s}^{-1}$ for the secondary star using eight $\mathrm{Mg}$, Ti, Fe, and Ce lines.

\section{Rotation and spectral variability}

\subsection{Spectral variability of the Ap component}

To search for spectral variability we used the separated spectra for each orbital phase, which is the same as the observed spectrum from which the mean reconstructed spectrum for one of the components has been subtracted. To combine HARPS and FEROS spectra, we degraded the HARPS resolution by convolving these spectra with a Gaussian of $7.5 \mathrm{~km} \mathrm{~s}^{-1}$ full-width at half-maximum, which is close to the FEROS instrumental profile. The secondary star presents strong spectral variability, which is clearly visible in $\mathrm{Fe}$ and rare-earth elements, despite the relatively low flux of the secondary component and hence lower effective signal-to-noise ratio $(\mathrm{S} / \mathrm{N})$ of its spectrum. The equivalent widths (EWs) of lines of many ions, such as Fe I-II, Ti II, Nd II-III, Sm II, and Tb II, have similar variations showing one-wave curves as a function of orbital phase. The phase of the highest intensity is different for the various elements, however, with Fe roughly opposite to Ti and rare-earth elements. We have plotted in Fig. 4 a small spectral region showing Fe and $\mathrm{Sm}$ variations. HARPS and FEROS spectra are labeled with orbital

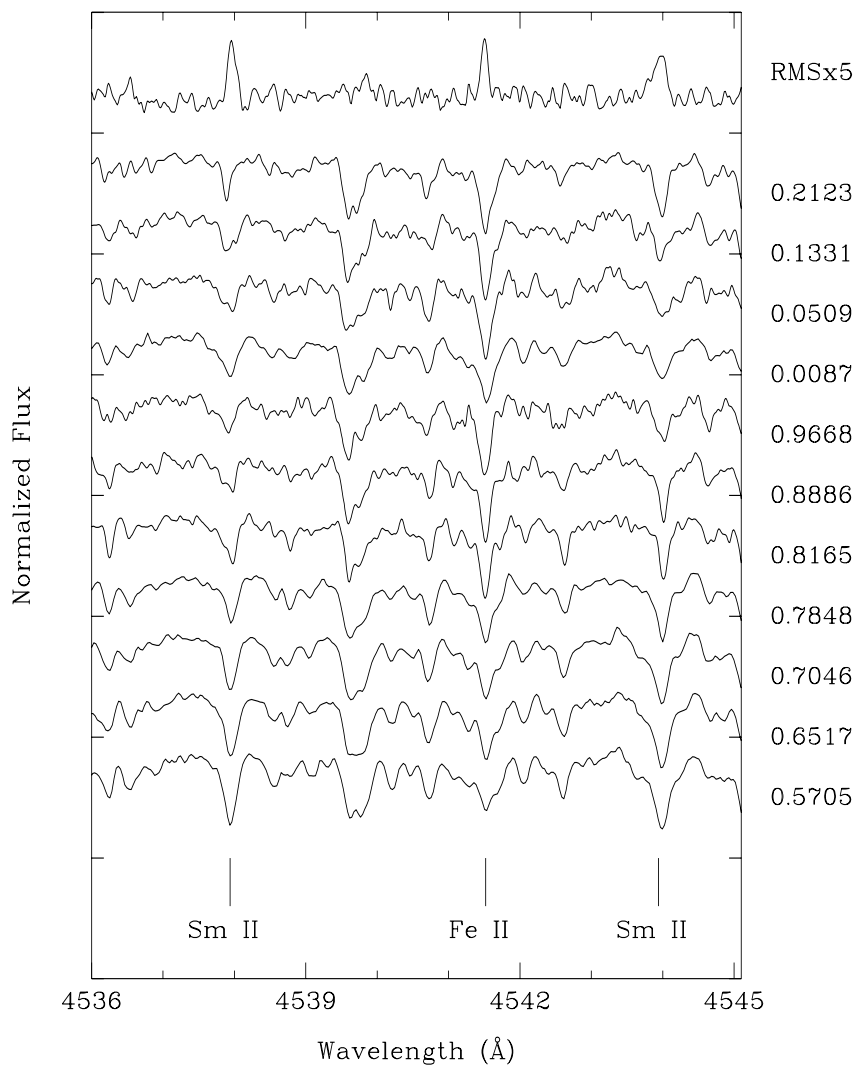

Fig. 4. Small spectral section showing the intensity line variations in the secondary Ap component. Ticks correspond to the spectral lines

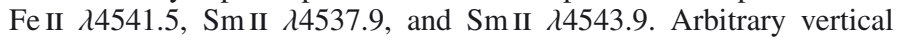
shifts have been applied for clarity. The ordinate scale is 0.1 per tick. Orbital phases are labeled at the right side, increasing from bottom to top. The first curve is the standard deviation of the residual of the 11 spectra with respect to the mean spectrum of the secondary, scaled by a factor of 5 for better visibility.

phase, while the first curve is the standard deviation of the residual of the 11 spectra with respect to the mean spectrum of the secondary. The two FEROS spectra taken at the same phase 0.57 have been combined into one in this figure.

Figure 5 shows the behavior of the spectral lines of various atomic species. Even though the number of spectra is not large, the acceptable phase coverage and the apparently simple shape of the EW curves allow us to estimate the rotation period, 


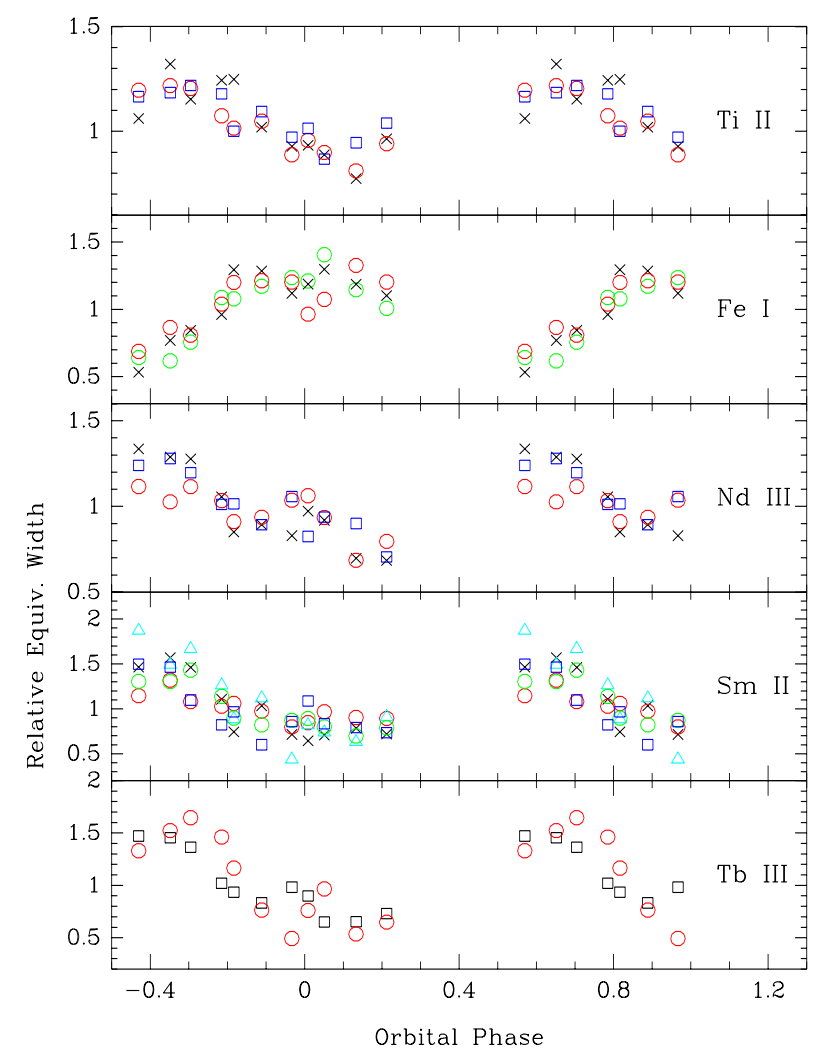

Fig. 5. Line intensity curves for various atomic species in the spectra of the secondary Ap component. Different symbols correspond to different lines. The lines plotted are Ti II $\lambda \lambda 4444,4780,4572$, Fe I $\lambda \lambda 5006,4476$, 4384, Nd III $\lambda \lambda 4990,4810,4788$, Sm II $\lambda \lambda 4453,4434,4704,4424$, 4538, and Tb III $\lambda \lambda 5505,5847$.

and hence to check the co-rotation hypothesis. With this aim we used EWs of several lines of Fe, Ti, Ce, Sm, and Nd. We applied the Lomb-Scargle method (Press \& Rybicki 1989) and found that the current observations are compatible with several different values of the rotational period, one of them very close to the orbital period, which is expected from the physical point of view, given the circularity of the orbit. More precisely, the mean rotational period, calculated from the variation of 21 spectral lines of $\mathrm{Fe}, \mathrm{Ti}, \mathrm{Ce}, \mathrm{Sm}$, and $\mathrm{Nd}$, is $P_{\text {rot }}=12.4490 \pm 0.0010$ days, while the typical uncertainty for the period derived from each individual line is about $0.004-0.005$ days.

This agrees excellently with the orbital period and eccentricity. In fact, for an orbital eccentricity in the range 0.0020-0.0066 (i.e. $\mathrm{a} \pm 1 \sigma$ interval), the pseudo-synchronization period (see e.g. Hut 1981) is in the range 12.4480-12.4509 days. Although small, the difference between the rotational and orbital period, $-0.0023 \pm 0.0010 \mathrm{~d}$, supports the finding that the orbital eccentricity is higher than zero.

Amplitudes of the EW variations are between $9 \%$ and $15 \%$ for the elements analyzed, while the phase of maximum abundance is 0.019 for Fe I, 0.629 for Ti II, and 0.604 on average for rare-earth elements. The region of the secondary-star surface that is permanently facing the primary star has the highest overabundance of rare-earth elements, while Fe is concentrated in the surface at the far side.

\subsection{Spectral variability of the HgMn component}

The strong spectral variations of the secondary star and its high line-density make it difficult to evaluate the variability of the primary star. Consequently, we selected strong spectral lines of various elements that were free of blends with lines of the secondary star in most phases. This analysis was made visually, and we considered an element to be variable only if several lines of the same element showed a similar behavior. As mentioned above, the spectral resolution of HARPS spectra was slightly degraded to make them comparable to FEROS spectra.

We found that $\mathrm{Mn}$ and $\mathrm{Cr}$ are variable in the primary star. Fig. 6 shows the variations of four $\mathrm{Cr}$ II $(\lambda \lambda$ 4242.36, 4824.13, 4848.24, 4876.41) and four Mn II ( $\lambda \lambda$ 4326.64, 4755.73, $4764.83,6122.434)$ lines. In this figure four HARPS spectra have been plotted corresponding to two different phases. To minimize the unreliability inherent in the secondary lines, we have omitted in these plots spectral regions where strong $(>1.5 \%$ of the binary continuum) secondary lines were located in the original composite spectra. It is clear from this figure that both $\mathrm{Mn}$ and $\mathrm{Cr}$ are stronger near the second quadrature $(\phi \approx 0.85)$ than near the first quadrature $(\phi \approx 0.17)$.

In Fig. 7 we show the statistical moments of three strong Mn lines in all FEROS and HARPS spectra. The similarity of the behavior of the three lines in various moments suggests the presence of a variation common to all $\mathrm{Mn}$ lines that is significantly larger than observational errors. The line profile variations detected in the spectrum of the primary star, however, are too weak to discuss its rotational period.

\subsection{Rotational velocity}

The projected rotational velocity that we used for deriving the stellar radius was measured with the technique of Díaz et al. (2011). This method determines $v \sin i$ through the first zero of the Fourier transform of the rotational profile, which is calculated from the cross-correlation function. It takes into account the limb-darkening effect and can be applied to spectral regions with line blends. The limb-darkening coefficient (linear law), which modifies the line profiles and is required for the calculation of $v \sin i$, was taken from Claret (2004). We used eleven spectral windows for the primary star, obtaining a mean value of $v \sin i=16.78 \mathrm{~km} \mathrm{~s}^{-1}$ with $\sigma=0.26 \mathrm{~km} \mathrm{~s}^{-1}$. This standard deviation, which is consistent with the measurement error of each spectral window, corresponds to the random noise and includes mainly the spectrum shot noise. A second contribution to the measurement error in this method is related with the spectraltype missmatch between object and template spectra. Although this error is largely included in the random error, we estimated its amount through experiments with different templates. We found that a deviation of $500 \mathrm{~K}$ in $T_{\text {eff }}$ and $0.5 \mathrm{dex}$ in $\log g$ causes a $v \sin i$ error of only $0.05 \mathrm{~km} \mathrm{~s}^{-1}$ each.

In addition to the formal measurement error, a realistic estimation of the rotational velocity should take into account the uncertainties related with the underlying assumption of the classic rotational model. Our measurement strategy considered the star as a rigidly rotating sphere of uniform surface brightness with a limb-darkening described by the standard linear law. The influence of line profile distortions caused by possible chemical spots in the star surface was evaluated by measuring the individual spectra taken at different orbital phases. We estimate that for the combination of all spectral lines this source of error is not important for the primary star, with a contribution probably lower than $0.1 \mathrm{~km} \mathrm{~s}^{-1}$. Naturally, spectral variability is not sensitive to axisymmetric spots, but the obtained global rotational profile does not show any significant deviation from the expected theoretical profile. Departures from sphericity and rigid rotation are expected to be negligible as well. The quotient of the first two 

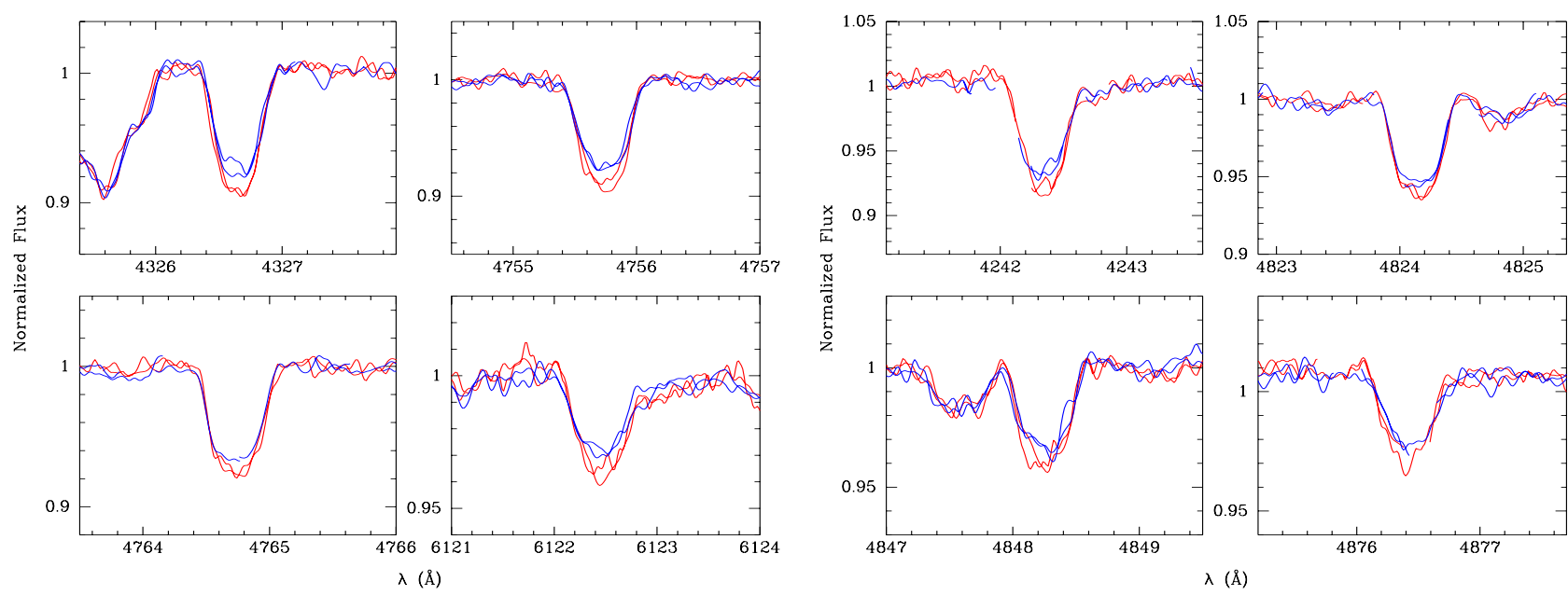

Fig. 6. Spectral variability in the primary $\mathrm{HgMn}$ star. Two spectra near phase 0.17 (blue) and two spectra near phase 0.85 (red) are plotted in the region of four Mn II lines (left panels) and four Cr II (right panels) lines.

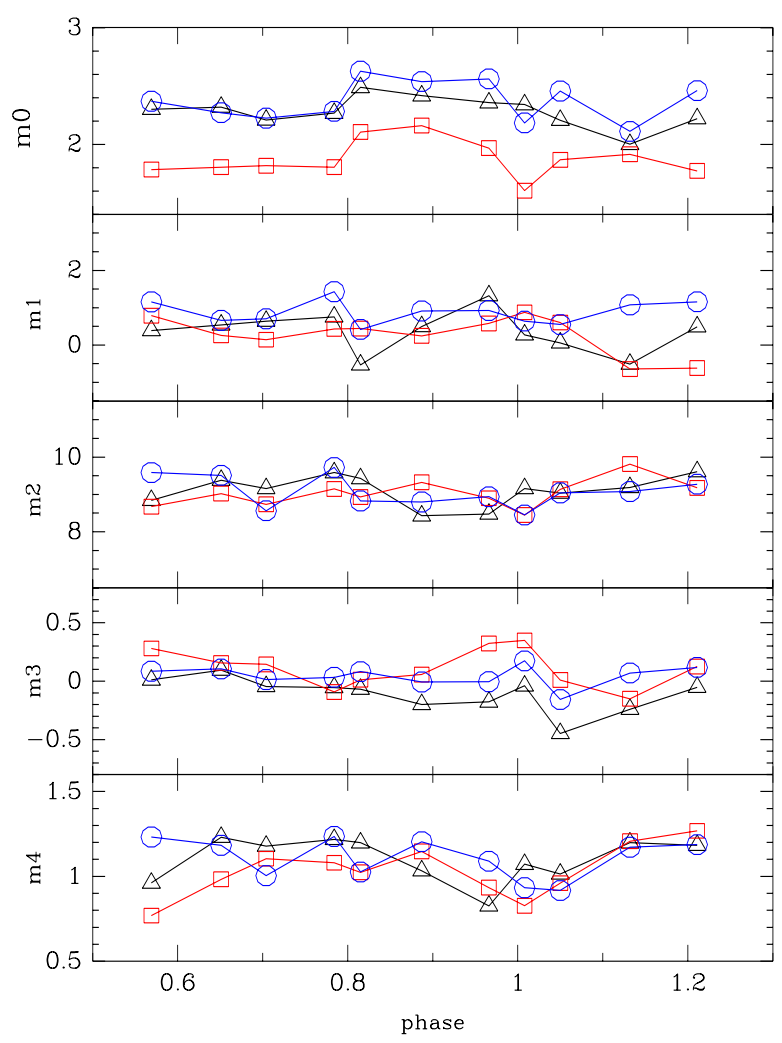

Fig. 7. Line profile variability for three strong Mn II lines in the primary HgMn star: $\lambda 4756$ (black triangles), $\lambda 4765$ (red squares), $\lambda 4327$ (blue circles). Statistical moments are plotted as a function of orbital phase: $\mathrm{m} 0=$ equivalent width, $\mathrm{m} 1=$ radial velocity, $\mathrm{m} 2=$ line width, $\mathrm{m} 3=$ asymmetry, $\mathrm{m} 4=$ kurtosis. Units are $\mathrm{km} \mathrm{s}^{-1}$ in the first (top) three panels, while $\mathrm{m} 4$ and $\mathrm{m} 5$ are dimensionless.

zeros of the Fourier transform of the rotational profile, which is a proxy for differential rotation (Reiners \& Schmitt 2002), is consistent with rigid rotation.

The combination of all error sources mentioned so far results in a standard error just over $1 \%$. The most important error source, however, is probably the one related with the fundamental assumption of the classic model: the line profile can be described as the convolution of the intrinsic line profile and a standard rotational profile, which is calculated with the limb-darkening coefficient corresponding to the continuum of that spectral region. The assumption that the limb darkening within the line profile equals that of the nearby continuum is equivalent to the assumption that the spectral morphology does not vary over the stellar disk. In fact, limb darkening would be in general weaker within the lines than in the continuum (Collins \& Truax 1995) and most likely does not average out by combining many lines of various intensities and formation depths. The differences between line and continuum limb-darkening can be as large as 0.2 for strong lines (Collins $\&$ Truax 1995), which would introduce an error on the order of $0.5 \mathrm{~km} \mathrm{~s}^{-1}$ in the projected rotational velocity. All in all, considering all contributions, we adopted for the primary star rotation $v_{\mathrm{A}} \sin i=16.8 \pm 0.6 \mathrm{~km} \mathrm{~s}^{-1}$.

The rotational profile of the secondary star is considerably narrower, and it is similar to the instrumental and the intrinsic line profiles. This makes the calculation of $v \sin i$ less accurate. On the other hand, the chemical spots distorting the rotational profiles are more notorious in this star, causing variations of about $10 \%$ in the apparent rotational velocity. The final value adopted for secondary star is $v_{\mathrm{B}} \sin i=7.6 \pm 1.1 \mathrm{~km} \mathrm{~s}^{-1}$.

The orbit of HD 161701 is nearly circular, even though the orbital period is relatively long. This suggests that an efficient circularization mechanism has been taking place in this system. The observed synchronization of the rotational motion of the secondary star with the orbit is assumed to be another consequence of that process.

In standard models for non-magnetic stars Claret (see e.g. 2004), in which tidal braking is driven by turbulent dissipation (dominant in stars with convective envelopes) or radiative damping (dominant in stars with radiative envelopes), the synchronization and circularization time scales for a binary with a 12 day period are much longer than their main-sequence lifetime. We speculate that large-scale magnetic fields may be the mechanism responsible for the high degree of circularization and synchronization reached by the system HD 161701 .

\section{Fundamental and atmospheric parameters}

Several independent sources are used in this section to obtain information on physical parameters of the stellar components. The spectroscopic orbit provides minimum masses $M_{\mathrm{A}} \sin ^{3} i$ and $M_{\mathrm{B}} \sin ^{3} i$, while the projected radius $R_{\mathrm{A}} \sin i$ is derived from the 


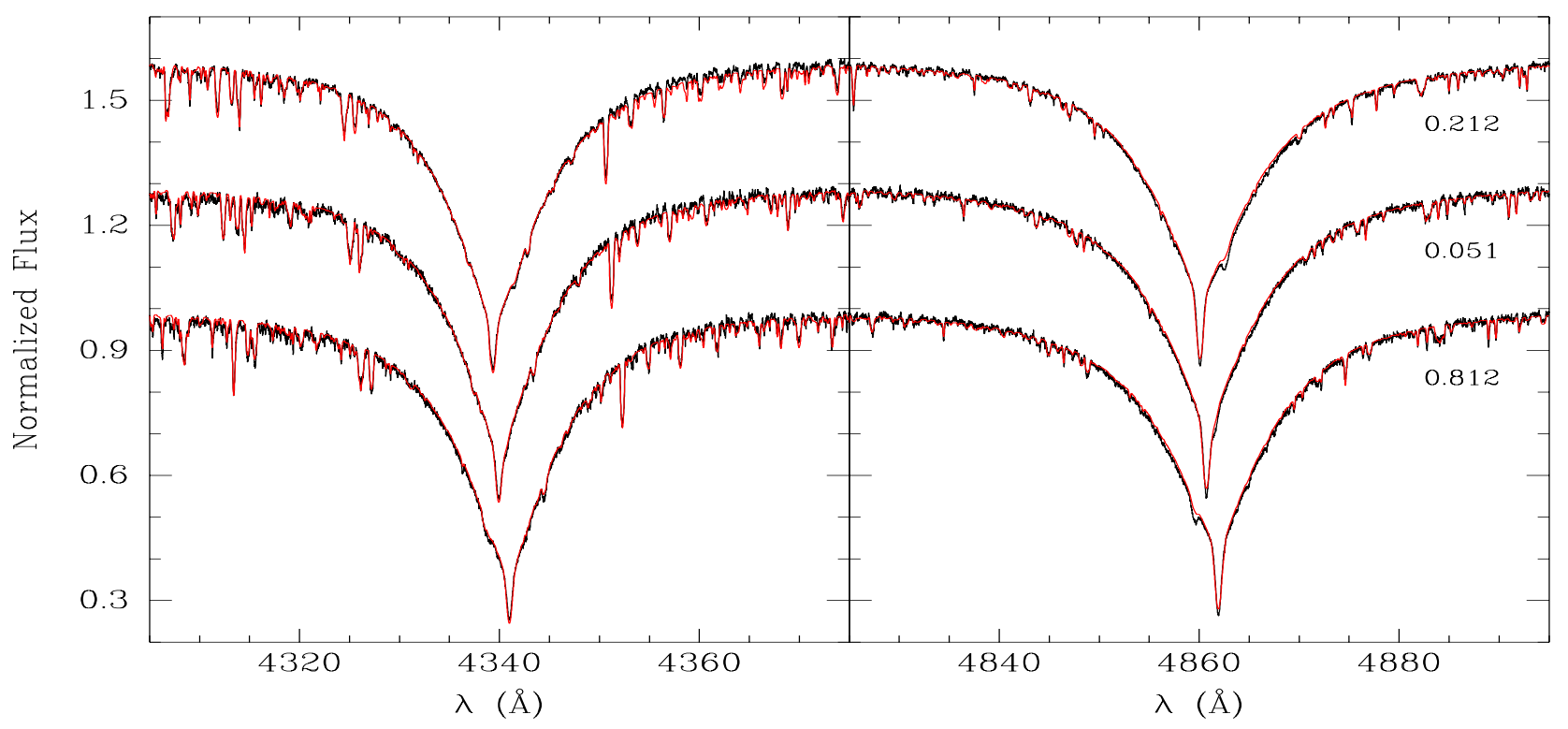

Fig. 8. Comparison of the observed HARPS (black) and synthetic (red) Balmer line profiles for $T_{\text {eff, } \mathrm{A}}=12400 \mathrm{~K}, \log g_{\mathrm{A}}=3.76$, and $T_{\text {eff, } \mathrm{B}}=$ $9750 \mathrm{~K}, \log g_{\mathrm{B}}=4.15$ at three orbital phases (labeled on the right).

rotational velocity, under the assumption of synchronization. On the other hand, synthesis of Hi line profiles as well as the requirement of ionization and excitation equilibria in $\mathrm{Fe}$ lines are used to get $T_{\text {eff }}$ and $\log g$. Finally, some restrictions on the stellar parameters are imposed using theoretical evolutionary models, assuming that both stars have the same age and requiring the masses to be compatible with the spectroscopic flux ratio $F_{\mathrm{B}} / F_{\mathrm{A}}$.

A preliminary estimate of temperature and surface gravity was derived from Geneva photometry by Lanz et al. (1993), obtaining $T_{\text {eff }}=12700 \mathrm{~K}$ and $\log g=4.15$. From Strömgren photometry we obtained $T_{\text {eff }}=12652 \pm 150 \mathrm{~K}$ and $\log g=$ $3.98 \pm 0.05$. In neither case the contribution from the secondary star was considered.

In a first step for deriving atmospheric parameters, we analyzed the profile of $\mathrm{H} \beta$ and $\mathrm{H} \gamma$ lines in the HARPS spectra. In $\mathrm{SB} 2 \mathrm{~s}$, fitting of $\mathrm{HI}$ line profiles is more uncertain than in single stars since broad spectral features, whose widths are larger than RV shifts, are poorly recovered by separating techniques. Furthermore, the flux contribution of each component to the continuum is in principle unknown. Therefore, for Balmer-line fitting we did not use separated spectra, but instead employed observed (composite) spectra taken at various orbital phases.

We calculated a grid of synthetic profiles for both components with a $50 \mathrm{~K}$ step in temperature and 0.05 step in $\log g$. To combine these profiles into a binary synthetic profile we calculated the radius ratio using the spectroscopic mass-ratio and the gravity values corresponding to the profiles to be combined:

$\left(\frac{R_{\mathrm{B}}}{R_{\mathrm{A}}}\right)^{2}=q \cdot 10^{\log g_{\mathrm{A}}-\log g_{\mathrm{B}}}$.

Synthetic binary profiles were then subtracted from observed spectra and the goodness of the solution was evaluated by the sum of squares of residuals and the highest absolute value of residuals. We estimate the uncertainty in the observed normalized flux to be on the order of 0.007 , based on the asymmetry of the $\mathrm{H} \beta$ profile and the difference between FEROS and HARPS spectra. We therefore considered as acceptable all synthetic profiles whose difference with the observed spectrum was lower than 0.007 in the spectral range 4825-4900 $(4305-$ $4475 \AA$ for $\mathrm{H} \gamma$ ), excluding strong metallic lines and the core of $\mathrm{H} \beta$, whose depth was more difficult to reproduce.

Configurations compatible with Balmer profiles are those with a primary temperature in the range $T_{\text {eff,A }}=12000$ $12800 \mathrm{~K}$ and $\log g_{\mathrm{A}}>3.82$. For the secondary star, the uncertainty in the flux ratio makes it much more difficult to derive its atmospheric parameters spectroscopically. We therefore decided to adopt for this star the temperature derived from the massradius diagram, as described below. A second fit of $\mathrm{H}$ I profiles was made after the abundance analysis, to use atmosphere models with appropriate chemical abundances for the synthesis of the Balmer lines. Line profiles for the adopted solution are shown in Fig. 8 for three different orbital phases at opposite quadratures and near conjunction.

In Fig. 9 the possible configurations of the binary are plotted in the mass-radius diagram. Filled and open symbols are the mass and radius of stellar components A and B for several values of the orbital inclination, which in principle is unknown. For each orbital inclination, masses were calculated from the spectroscopic minimum masses $M \sin ^{3} i$. Projected stellar radii $R \sin i$ were calculated from $v \sin i$ assuming that for both stars the rotational period equals the orbital period and that the rotational axis are perpendicular to the orbital plane. In Sect. 5 we derived the projected rotational velocities and discussed the rotational period. For the secondary component we have determined observationally the rotational period, but the $v \sin i$ value is rather uncertain. In contrast, for the primary star we obtained a more accurate projected rotational velocity, but we do not have independent information on its rotational period. We assume here that both stars are pseudo-synchronized with the orbital motion to calculate the projected stellar radii which, in combination with orbital parameters, provides the relative radii without an assumption about the orbital inclination: $R_{\mathrm{A}} / a=0.104 \pm 0.004$, $R_{\mathrm{B}} / a=0.047 \pm 0.015$.

We took as an additional constraint that temperature, mass, and radius of the components have to be consistent with two main-sequence stars of the same age. With this aim we interpolated in the evolutionary models of the Geneva group (Schaller et al. 1992; Mowlavi et al. 2012), the mass and radius 


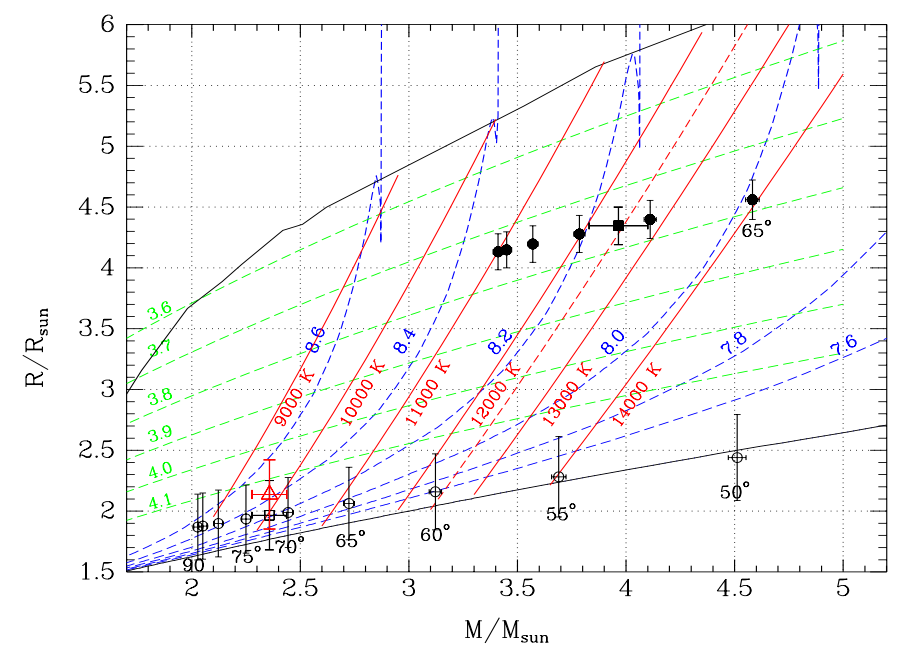

Fig. 9. Mass-radius diagram. Filled and open circles are the position of the primary and secondary star for different orbital inclinations, decreasing from $90^{\circ}$ with a step of $5^{\circ}$. Squares correspond to the solution with orbital inclination $i=72 \mathrm{deg}$ and the open triangle to the secondary configuration with $\log g=4.15$. Various theoretical sequences have been plotted for comparison: isochrones (dashed blue lines, labeled with $\log ($ age) $)$, zero-age, and terminal-age main sequences (black), isotherms (red), and iso-gravity curves (green, labeled with $\log g$ ).

corresponding to $T_{\text {eff }}$ and $\log g$ values of both companions in every configuration in our profile grid. In Fig. 9 isochrones, isotherms, and iso-gravity curves corresponding to Geneva stellar models have been plotted.

The temperature of the primary constrains the possible values of orbital inclination to the interval $69-74^{\circ}$. Error bars plotted to the solution corresponding to $i=72 \mathrm{deg}$ (square symbols) in Fig. 9 correspond to this range, that is, they include the temperature uncertainty. The good precision of the rotational velocity of the primary $(3.6 \%)$ clearly defines the surface gravity of this star: assuming an inclination $i=72 \pm 2^{\circ}$, we obtain $\log g_{\mathrm{A}}=3.762 \pm 0.031$.

Note that rotational velocities are consistent with the assumption that both stars are normal stars of the same age. More precisely, the age of the primary and the rotational velocity of the secondary both suggest that the secondary star has a small radius, with $\log g_{\mathrm{B}}=4.10-4.35$. This star would not be very close to the zero-age main-sequence, however. Solutions with very low flux ratios are not consistent with the intensity of secondary metallic lines in the spectrum. In fact, strong metallic lines of the secondary star, such as Mg II $\lambda 4481$, Fe II $\lambda 4549$, and Fe II 25169 , have depths of about 0.09 in the composite spectrum, which implies that the flux contribution of the secondary star is at least $0.12-0.13$ of the total light, suggesting $\log g_{\mathrm{B}} \lesssim 4.15$. We therefore adopted this value to calculate the chemical abundances with spectral synthesis, allowing a better fitting of the spectrum. This small difference in $\log g_{\mathrm{B}}$ is within observational uncertainties, however, and we consider it not enough to discard either the co-rotation hypothesis or the age agreement between the two companions. Finally, during the abundance calculations, the ionization and excitation equilibrium of $\mathrm{Fe}$ lines were used to refine the atmospheric parameters. The final fundamental parameters adopted for both stellar components are listed in Table 4.
Table 4. Physical parameters, calculated after adopting an orbital inclination of $i=72.0 \pm 2^{\circ}$.

\begin{tabular}{lc}
\hline \hline$a\left[R_{\odot}\right]$ & $41.8 \pm 0.1$ \\
$R_{\mathrm{A}}\left[R_{\odot}\right]$ & $4.34 \pm 0.16$ \\
$R_{\mathrm{B}}\left[R_{\odot}\right]$ & $2.14 \pm 0.28$ \\
$M_{\mathrm{A}}\left[M_{\odot}\right]$ & $3.96 \pm 0.14$ \\
$M_{\mathrm{B}}\left[M_{\odot}\right]$ & $2.36 \pm 0.08$ \\
$\log g_{\mathrm{A}}$ & $3.76 \pm 0.03$ \\
$\log g_{\mathrm{B}}$ & $4.15 \pm 0.13$ \\
$T_{\text {eff, } \mathrm{A}}[\mathrm{K}]$ & $12400 \pm 300$ \\
$T_{\text {eff, } \mathrm{B}}[\mathrm{K}]$ & $9750 \pm 300$ \\
$\log L_{\mathrm{A}} / L_{\odot}$ & $2.61 \pm 0.06$ \\
$\log L_{\mathrm{B}} / L_{\odot}$ & $1.57 \pm 0.20$ \\
\hline
\end{tabular}

\section{Chemical abundances}

The abundance analysis was based on the separated HARPS spectra for stars A and B. These mean spectra are expected to reflect the average surface properties of the stars over the orbital cycle and not at some individual phase. Since the continuum intensity of the separated spectra is in principle undetermined, the relative flux contribution of each component to the binary continuum was calculated from the radius ratio and the surface energy flux distribution. Using the adopted atmospheric paramters and the calculated abundances (these calculations were performed iteratively), we computed the surface flux spectra $f_{\mathrm{A}}$ and $f_{\mathrm{B}}$ with the SYNTHE code. Then we calculated the relative contribution of stars $\mathrm{A}$ and $\mathrm{B}$ to the continuum, $c_{\mathrm{A}}$ and $c_{\mathrm{B}}$ respectively, from the relations $c_{\mathrm{B}} / c_{\mathrm{A}}=\left(f_{\mathrm{B}} / f_{\mathrm{A}}\right) \cdot\left(R_{\mathrm{B}} / R_{\mathrm{A}}\right)^{2}$ and $c_{\mathrm{B}}+c_{\mathrm{A}}=1$. These continuum spectra $c_{\mathrm{A}}$ and $c_{\mathrm{B}}$ were used to scale the spectra of both components to recover the intrinsic intensity of their spectral lines.

We derived the chemical abundances by using synthetic spectra calculated with the Linux version (Sbordone et al. 2004) of the SYNTHE code (Kurucz 1993b) and with an ATLAS9 (Kurucz 1993a) model atmosphere.

We adopted atmospheric parameters determined in the present study (see Table 4 ) for the primary $\left(T_{\text {eff,A }}=12400 \mathrm{~K}\right.$, $\left.\log g_{\mathrm{A}}=3.76\right)$ and for the secondary $\left(T_{\mathrm{eff}, \mathrm{B}}=9750 \mathrm{~K}, \log g_{\mathrm{B}}=\right.$ 4.15). A zero microturbulent velocity was adopted for both stars. Synthetic spectra were rotationally broadened using the $v \sin i$ values obtained in Sect. 5.3, that is, $16.9 \mathrm{~km} \mathrm{~s}^{-1}$ and $7.0 \mathrm{~km} \mathrm{~s}^{-1}$, for stars A and B, respectively. We adopted a Gaussian instrumental profile with a resolving power of 110000 , corresponding to the HARPS spectrograph.

The atomic line list and $\log g f$ data used in this work is basically the one described in Castelli \& Hubrig (2004) ${ }^{1}$. The updated version of the line list includes the Fuhr \& Wiese (2006) $\log g f$ data for several Fe I and Fe II lines. Some specialized references have also been added for different elements, such as Dy I-II (Wickliffe et al. 2000), Eu II (Lawler et al. 2001a), Nd II (Den Hartog et al. 2003), Tb II (Lawler et al. 2001b), La II (Lawler et al. 2001c), Gd II (Den Hartog et al. 2006), Sm II (Lawler et al. 2006), Ce II (Lawler et al. 2009), and rare-earths DREAM database $^{2}$ (Dy III, Er II-III, Eu III, Ho III, Nd III, Pr II-III, Tb III).

We started the calculations using an ATLAS9 model atmosphere with solar abundance values (Grevesse et al. 1996). The abundances in the synthetic spectrum were then modified to obtain agreement between the observed and computed profiles of

\footnotetext{
Available online at

http://wwwuser. oat.ts.astro.it/castelli/linelists.html

2 http://w3.umons.ac.be/ astro/dream.shtml
} 
Table 5. Chemical abundances $\log \left(N / N_{\text {tot }}\right)$.

\begin{tabular}{|c|c|c|c|c|c|c|c|c|c|}
\hline \multirow[t]{2}{*}{ Ion } & \multicolumn{4}{|c|}{ Star A } & \multicolumn{4}{|c|}{ Star B } & \multirow[t]{2}{*}{ Sun } \\
\hline & Mean & $\sigma$ & $n$ & err & Mean & $\sigma$ & $n$ & err & \\
\hline $\mathrm{He} \mathrm{I}$ & -2.46 & 0.21 & 4 & 0.18 & & & & & -1.05 \\
\hline C II & -3.86 & 0.04 & 3 & 0.15 & & & & & -3.52 \\
\hline O I & -3.63 & 0.11 & 4 & 0.06 & & & & & -3.21 \\
\hline $\mathrm{Na} I$ & & & & & -5.17 & 0.17 & 2 & 0.57 & -5.71 \\
\hline $\mathrm{Mg}_{\mathrm{I}}$ & -5.75 & & 1 & 0.31 & -4.08 & 0.25 & 5 & 0.47 & -4.46 \\
\hline $\mathrm{Mg}_{\text {II }}$ & -5.32 & 0.05 & 4 & 0.07 & -4.50 & 0.16 & 4 & 0.32 & -4.46 \\
\hline $\mathrm{Al}$ II & -7.78 & & 1 & 0.17 & & & & & -5.57 \\
\hline Si II & -4.38 & 0.13 & 10 & 0.08 & -5.44 & 0.17 & 4 & 0.32 & -4.49 \\
\hline $\mathrm{P}_{\mathrm{II}}$ & -5.08 & 0.05 & 3 & 0.10 & $-4.9:$ & & 1 & 0.43 & -6.59 \\
\hline S II & -5.92 & 0.01 & 2 & 0.12 & & & & & -4.71 \\
\hline $\mathrm{Ca} I$ & & & & & -5.51 & 0.41 & 2 & 0.38 & -5.68 \\
\hline Ca II & -5.95 & & 1 & 0.17 & -5.50 & 0.17 & 3 & 0.28 & -5.68 \\
\hline Sc II & & & & & -9.72 & & 1 & 0.30 & -8.87 \\
\hline Ti II & -6.36 & 0.08 & 43 & 0.13 & -6.03 & 0.20 & 52 & 0.51 & -7.02 \\
\hline $\mathrm{Cr} \mathrm{I}$ & & & & & -4.74 & 0.17 & 7 & 0.49 & -6.37 \\
\hline Cr II & -6.46 & 0.08 & 18 & 0.08 & -4.92 & 0.21 & 39 & 0.47 & -6.37 \\
\hline Mn I & & & & & -5.64 & 0.20 & 13 & 0.28 & -6.65 \\
\hline Mn II & -5.41 & 0.16 & 29 & 0.07 & -5.37 & 0.17 & 11 & 0.26 & -6.65 \\
\hline $\mathrm{Fe} \mathrm{I}$ & -3.85 & 0.09 & 32 & 0.11 & -3.54 & 0.24 & 94 & 0.35 & -4.54 \\
\hline $\mathrm{Fe}$ II & -3.89 & 0.09 & 117 & 0.05 & -3.44 & 0.20 & 104 & 0.41 & -4.54 \\
\hline $\mathrm{Fe}$ III & -3.98 & & 1 & 0.15 & & & & & -4.54 \\
\hline Ni II & -7.64 & & 1 & 0.17 & & & & & -5.79 \\
\hline Ga II & -5.92 & 0.21 & 5 & 0.11 & & & & & -9.16 \\
\hline Sr II & -8.29 & 0.03 & 2 & 0.14 & -6.44 & 0.22 & 4 & 0.46 & -9.07 \\
\hline Y II & -7.82 & 0.09 & 8 & 0.12 & -7.74 & 0.19 & 14 & 0.43 & -9.80 \\
\hline Zr II & -8.44 & & 1 & 0.10 & -8.09 & 0.30 & 7 & 0.27 & -9.44 \\
\hline Xe II & -5.87 & 0.26 & 8 & 0.12 & & & & & -9.87 \\
\hline La II & & & & & -8.94 & 0.51 & 2 & 0.37 & -10.87 \\
\hline Ce II & & & & & -8.07 & 0.15 & 14 & 0.27 & -10.46 \\
\hline Pr II & & & & & -8.02 & 0.51 & 2 & & -11.33 \\
\hline Pr III & & & & & -7.14 & 0.52 & 11 & 0.46 & -11.33 \\
\hline Nd II & & & & & -7.22 & 0.24 & 21 & 0.32 & -10.54 \\
\hline Nd III & & & & & -6.19 & 0.41 & 23 & 0.55 & -10.54 \\
\hline Sm II & & & & & -7.79 & 0.23 & 13 & 0.23 & -11.03 \\
\hline Eu II & & & & & -8.26 & 0.26 & 2 & 0.37 & -11.53 \\
\hline Tb III & & & & & -8.18 & 0.61 & 3 & 0.40 & -11.69 \\
\hline Dy II & & & & & -8.90 & 0.72 & 7 & 0.38 & -10.90 \\
\hline Dy III & & & & & -7.19 & 0.21 & 8 & 0.23 & -10.90 \\
\hline Ho III & & & & & -7.72 & & 1 & 1.26 & -11.78 \\
\hline Er II & & & & & -8.61 & 0.03 & 3 & 0.25 & -11.11 \\
\hline Er III & & & & & -6.92 & 0.06 & 2 & 0.30 & -11.11 \\
\hline Tm II & & & & & -7.80 & 0.52 & 2 & 0.41 & -12.00 \\
\hline Hg II & -6.76 & & 1 & 0.11 & & & & & -10.91 \\
\hline
\end{tabular}

Notes. For each star and atomic species the mean value, the standard deviation, the number of lines, and the adopted error are listed.

selected lines. For this comparison we calculated and minimized the quadratic sum of the differences between both spectra in the line neighborhood. In the final abundance calculations the model atmospheres ware calculated with appropriate abundance values and a final synthetic spectrum was produced.

The final adopted stellar abundances for the primary and secondary stars are listed in Table 5. We have included the number of lines involved in the average and the standard deviation. The error consigned in the table is the quadratic sum of two contributions: the measurement error and the error related to the atmospheric parameters uncertainty.

The measurement random error, estimated from line-to-line dispersion, is related mainly to the spectrum photon noise, blends with faint lines, and eventually errors in line parameters $(\log g f)$. It was calculated as $\max (\epsilon, \sigma) / \sqrt{n}$, where $\sigma^{2}$ is the variance of the line-by-line abundances of that element, $n$ is the number of lines, and $\epsilon$ is the typical error for an individual line.
The value of $\epsilon$ was estimated from the variance of line abundance in elements with many $(n>8)$ lines, obtaining for star A $\epsilon=0.10 \mathrm{dex}$ and for star B $0.21 \mathrm{dex}$ for elements with $Z<40$ and 0.37 for rare-earth elements. This error definition allowed us to assign measurement errors for elements with one or very few lines.

To evaluate the contribution to the abundance error of the uncertainties in the atmospheric and stellar parameters, we recalculated all abundances with modified values of $T_{\text {eff }}, \log g$, and $v_{\text {turb. }}$. The light-ratio factor was introduced in these calculations as a function of atmospheric parameters and mass-ratio: $\left(f_{\mathrm{B}} / f_{\mathrm{A}}\right) \cdot q \cdot 10^{\log g_{\mathrm{A}}-\log g_{\mathrm{B}}}$. In this manner, the abundance error of one component also depends on the atmospheric parameters of its companion through the relative flux ratio.

For star A this error was typically $0.05-0.10 \mathrm{dex}$; the main contribution is the uncertainty in $T_{\text {eff,A. In the secondary star the }}$ light-ratio strongly affects the line intensity, and consequently 

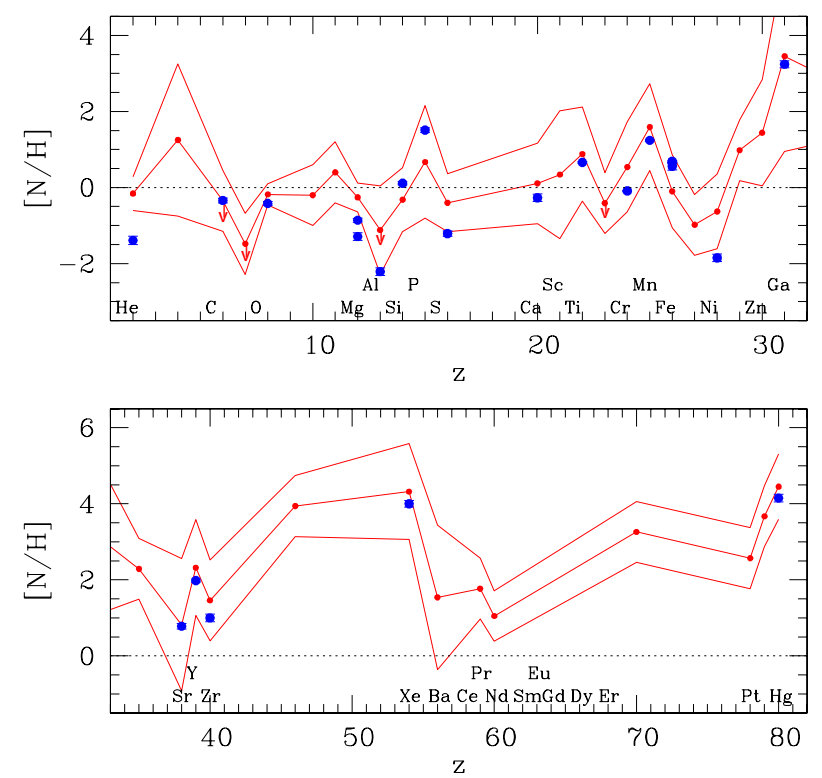

Fig. 10. Abundance pattern of the primary star (big blue circles) compared with the mean pattern of $\mathrm{HgMn}$ stars (small red circles and lines). Two additional lines mark intervals of $\pm 2 \sigma$ around the mean value.

the main error contribution is related to $\log g_{\mathrm{B}}$, that is, to the secondary radius.

Tables 6 and 7 (available at the CDS) list the line-by-line abundances for stars A and B, respectively. For each stellar companion a plot of the synthetic spectrum for the adopted chemical abundances along with the separated HARPS and the FEROS spectra is provided (available at the CDS).

To compare the derived abundances with the typical chemical pattern of chemically peculiar star groups, we present in Figs. 10 and 11 plots of abundance (relative to the Sun) vs atomic number. Star A is compared with the mean chemical pattern of $\mathrm{HgMn}$ stars, and star B with Am and ApSi stars. Small red points and continuous lines represent the average abundance and standard deviation of chemical peculair stars, while big blue circles correspond to the values found for HD 161701.

For the HgMn values shown in Fig. 10 we used the average of $11 \mathrm{HgMn}$ stars studied by different authors (Frémat \& Houziaux 1997; Castelli \& Hubrig 2004; Adelman et al. 2006; Catanzaro \& Leone 2006; Zavala et al. 2007; Adelman $\&$ Yüce 2010). For the $\mathrm{Ap}(\mathrm{Si})$ pattern we averaged five stars taken from compatible sources of literature (López-García et al. 2001; Albacete-Colombo et al. 2002; López-García \& Adelman 1999, 1994), and for the Am pattern five stars from Adelman et al. (1997); Adelman \& Albayrak (1998); and Adelman et al. (1999).

Star A has a chemical pattern typical of HgMn stars with notable overabundances of $\mathrm{P}, \mathrm{Mn}, \mathrm{Ga}, \mathrm{Y}, \mathrm{Xe}$, and Hg. Star B appears to be a classical Ap star, with overabundances of 1-2 dex in $\mathrm{Ti}, \mathrm{Cr}, \mathrm{Mn}$, and $\mathrm{Fe}$, of 2-3 dex in $\mathrm{Sr}$ and $\mathrm{Y}$, and 2.5-5 dex in all rare-earth elements. We noted that spectral lines of some elements are strongly variable and the assigned abundances should be taken as indicative of the average value over the rotational phases observed with HARPS.

\section{Discussion}

All observational information analyzed in Sect. 6 is consistent with a binary system consisting of two main-sequence stars of
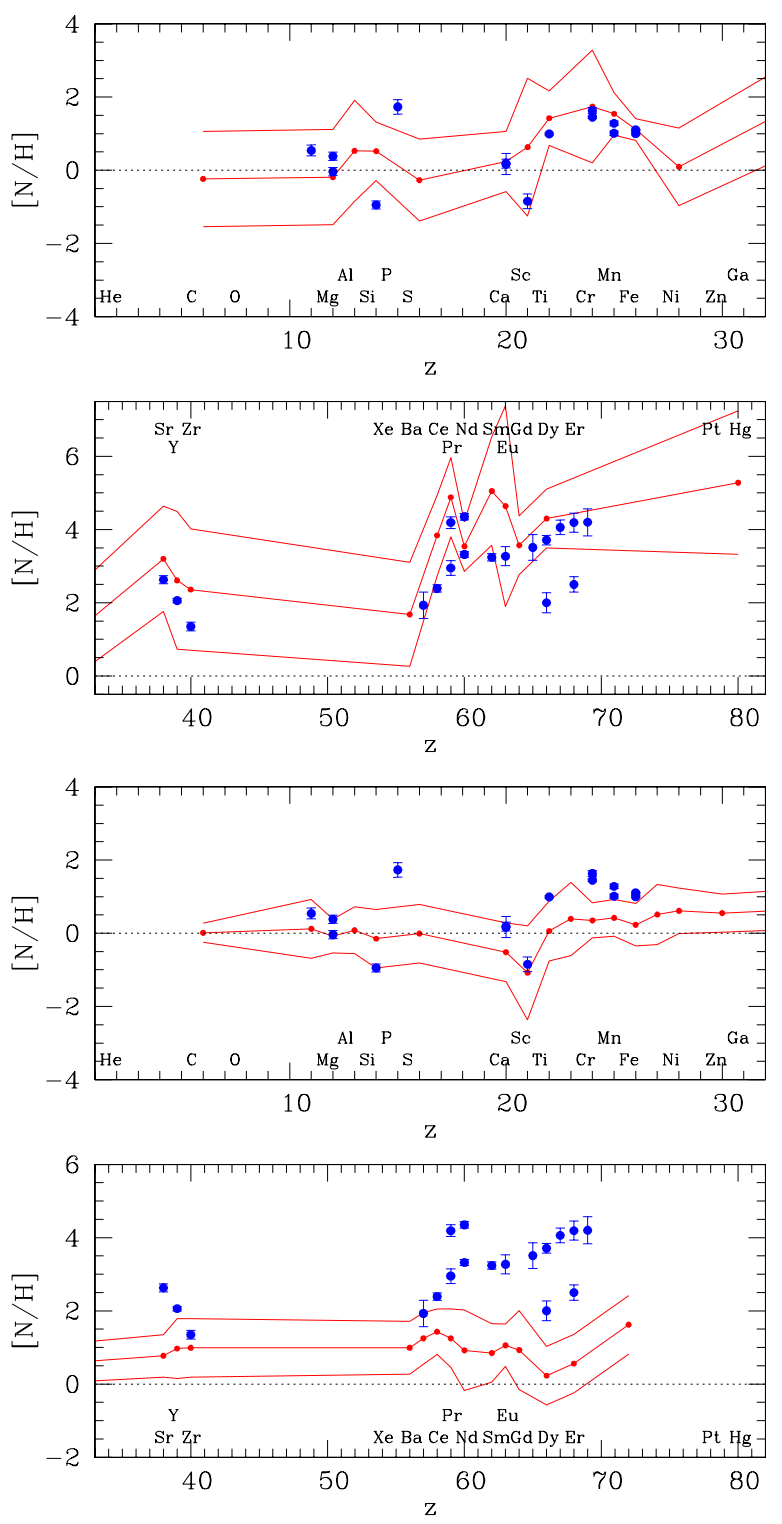

Fig. 11. Abundance pattern of the secondary star (big blue circles) compared with the mean pattern of ApSi stars (upper panels) and Am stars (lower panels).

age $\log \tau=8.15$. This is a typical age for HgMn stars. In the $\mathrm{WEBDA}^{3}$ database most clusters with stars mentioned to have spectra with strong $\mathrm{Hg}$ and/or Mn lines are in the age range $\log \tau=8.0-8.7$. However, there exist a few HgMn stars that are close to the zero-age main-sequence, as can be deduced from their stellar parameters or from their membership to very young stellar associations. This is the case of AR Aur (Woolf \& Lambert 1999), HR 6000 (Castelli \& Hubrig 2007), AO Vel CD (González et al. 2006), HD 37866, and BD -00 984 (Woolf \& Lambert 1999), and possibly 41 Eri, $\beta$ Scl, 66 EriB, and HD 221507 (Hubrig et al. 2012).

In binary systems with a $\mathrm{HgMn}$ star, the companion often also exhibits some kind of chemical peculiarity. Among binaries with stars of similar masses, several systems with both

3 The WEBDA database (http://www.univie.ac.at/webda/ webda.html), originally developed by J.-C. Mermilliod, is maintained by E. Paunzen and Christian Stütz, and is operated at the Institute for Astronomy of the University of Vienna. 
components of the HgMn type have been detected (e.g. 41 Eri, HR 7694, 46 Dra, HD 33647), while in systems with lower mass-ratio, secondaries with characteristics similar to Am stars have been reported (AR Aur, $\xi$ Lup, HR 4072, 112 Her). To our knowledge, this would be the first binary with a HgMn primary and a classical Ap companion. A multiple star in which a classical Bp star coexists with HgMn companions is the interesting quadruple system AO Vel, in which a close pair of two HgMn stars are bound to a more massive binary whose primary is a hotter BpSi star (González et al. 2006, 2010). Naturally, the detection of binaries with cool Ap or Am stars as companions of HgMn stars has often been hindered by the relatively low luminosity of the companion. Several known low mass-ratio $(q<$ 0.5) HgMn SB2s have not been subject of spectral separation and detailed abundance analysis (e.g. $\kappa C n c$, HD 124740, $\iota \mathrm{CrB}$, HR 6520, AV Acl Dolk et al. 2003). Finally, some open clusters with extensive spectroscopic studies are known to harbor peculiar stars of different classes. Both NGC 2516 and NGC 2287, for example, contain several $\mathrm{HgMn}, \mathrm{Ap}-\mathrm{BpSi}$, and Am stars.

A very interesting, but still little studied question is the possible connection of chemical anomalies with binarity. A phenomenological connection of chemical spots with the relative position of the binary companion has been previously demonstrated in a few SB2 HgMn stars. AR Aur, 41 Eri, and 66 Eri are three SB2 with $\mathrm{HgMn}$ components showing strong spectral variability. The most notable chemical spots have been found for the elements $\mathrm{Sr}$ and $\mathrm{Y}$ in $\mathrm{AR}$ Aur $\mathrm{A}, \mathrm{Mn}, \mathrm{Hg}$, and $\mathrm{Ga}$ in 41 Eri B (Hubrig et al. 2006, 2012), and Sr, Ba, and Y in 66 Eri A (Makaganiuk et al. 2011). Strikingly, in all these cases the lowerabundance surface region is the one facing the companion star, while the strong overabundance spots are located at the far side.

For HD 161701 the surface distribution of abnormal chemical elements in the HgMn companion is not clear, mainly because spectral line variations, although clear, are not strong. But in the secondary star, the overabundance of the elements with the most asymmetric distribution is clearly affected by the presence of the companion. The portion of the secondary star that is permanently facing the primary star has the highest abundance of rare-earth elements and $\mathrm{Ti}$, while $\mathrm{Fe}$ is concentrated in the surface region at the far side.

The cause of this fact is not known but it might be related with a modification of atmospheric conditions $\left(T_{\mathrm{eff}}, g\right)$ by the radiation and gravitational field of the companion, or by the magnetic-field structure. The long period of HD 161701 makes the mutual influence relatively moderate. Tidal forces are expected to cause gravity variations over the surface on the order of $\Delta \log g \approx 0.003-0.004$ dex for both components. Temperature variations due to a reflexion effect would be about $0.6 \%(60 \mathrm{~K})$ for the secondary companion, but negligible $(<5 \mathrm{~K})$ for the more luminous primary star. On the other hand, the magnetic topology has also to be considered as a possible cause of chemical spots. In fact, according to Hubrig et al. (in prep.), the secondary star has a relatively strong and variable magnetic field on the order of $200 \mathrm{G}$, whose global topology shows a clear correlation with the surface chemical pattern.

Acknowledgements. This work was supported in part by the Deutsche Forschungsgemeinschaft (Hu532/17-1) and by the ANPCYT of Argentina (PICTO-09/125).

\section{References}

Abt, H. A., Conti, P. S., Deutsch, A. J., \& Wallerstein, G. 1968, ApJ, 153, 177 Adelman, S. J., \& Albayrak, B. 1998, MNRAS, 300, 359
Adelman, S. J. \& Yüce, K. 2010, Astron. Nachr., 331, 785

Adelman, S. J., Caliskan, H., Kocer, D., \& Bolcal, C. 1997, MNRAS, 288, 470

Adelman, S. J., Caliskan, H., Cay, T., Kocer, D., \& Tektanali, H. G. 1999, MNRAS, 305, 591

Adelman, S., Caliskan, H., Gulliver, A. F., \& Teker, A. 2006, A\&A, 447, 685

Aikman, G. C. L. 1976, PDAO, 14, 379

Albacete-Colombo, J. F., López-García, Z., Levato, H., Malaroda, S. M., \& Grosso, M. 2002, A\&A, 392, 613

Babcock, H. W. 1958, ApJS, 3, 141

Biémont, E., Palmeri, P., \& Quinet, P. 1999, Ap\&SS, 269, 635

Black, J. H., Wisheit, J. C., \& Laviana, E. 1972, ApJ, 177, 567

Carrier, F., North, P., Udry, S., \& Babel, J. 2002, A\&A, 394, 151

Castelli, F., \& Hubrig, S. 2004, A\&A, 425, 263

Castelli, F., \& Hubrig, S. 2007, A\&A, 475, 1041

Catanzaro, G., \& Leone, F. 2006, MNRAS, 373, 330

Claret, A. 2004, A\&A, 428, 1001

Collins, G. W., II, \& Truax, R. J. 1995, ApJ, 439, 860

Cowley, C. R., \& Corliss, C. H. 1983, MNRAS, 203, 651

Cowley, A. P., Cowley, C. R., Hiltner, W. A., et al. 1968, PASP, 80, 746

Cowley, A., Cowley, C., Jaschek, M., \& Jaschek, C. 1969, AJ, 74, 375

Den Hartog, E. A., Lawler, J. E., Sneden, C., \& Cowan, J. J. 2003, ApJS, 148, 543

Den Hartog, E. A., Lawler, J. E., Sneden, C., \& Cowan, J. J. 2006, ApJS, 167, 292

Díaz, C. G., González, J. F., Levato, H., \& Grosso, M. 2011, A\&A, 531, A143

Dolk, L., Walgren, G. M., Hubrig, S. 2003, A\&A, 402, 299

Dworetsky, M. M., Jomaron, C. M., \& Smith, C. A. 1998, A\&A, 333, 665

Frémat, Y., \& Houziaux, L. 1997, A\&AS, 320, 580

Fuhr, J. R., \& Wiese, W. L. 2006, J. Phys. Chem. Ref. Data, 35, 4

Fuhr, J. R., Martin, G. A., \& Wiese, W. L. 1988, J. Phys. Chem. Ref. Data, 17, 4

González, J. F., \& Levato, H. 2006, A\&A, 448, 283

González, J. F., Hubrig, S., Nesvacil, N., \& North, P. 2006, A\&AS, 449, 327

González, J. F., Hubrig, S., \& Castelli, F. 2010, MNRAS, 402, 2539

Grevesse, N., Noels, A., \& Sauval, A. 1996, Proc. sixth annual October Astrophysics Conference in College Park, eds. Stephen S. Holt, \& George Sonneborn, ASP Conf. Ser., 99, 117

Hannaford, P., Lowe, R. M., Grevesse, N., Bimont, E., \& Whaling, W. 1982, ApJ, 261, 736

Hibbert, A. 1988, Phys. Scr., 38, 37

Hube, D. P. 1969, J. R. Astron. Soc. Can., 63, 229

Hubrig, S., North, P., \& Mathys, G. 2000, ApJ, 539, 352

Hubrig, S., González, J. F., Savaov, I., et al. 2006, MNRAS, 371, 1953

Hubrig, S., Savaov, I., Ilyin, I., et al. 2010, MNRAS, 408, L61

Hubrig, S., González, J. F., Ilyin, I., et al. 2011, Astron. Nachr., 332, 998

Hubrig, S., González, J. F., Ilyin, I., et al. 2012, A\&A, 547, A90

Hut, P. 1981, A\&A, 99, 126

Kling, R., Schnabel, R., \& Griesmann, U. 2001, ApJS, 134, 173

Kurucz, R. L. 1988, Trans. IAU, XXB, ed. M. McNally (Dordrecht: Kluwer), 168

Kurucz, R. 1993a, ATLAS9 Stellar Atmosphere Programs and $2 \mathrm{~km} \mathrm{~s}^{-1}$ grid, CD-ROM, No. 13

Kurucz, R. 1993b, SYNTHE Spectrum Synthesis Programs and Line Data, CD-ROM, No. 18 (Cambridge, MA: Smithsonian Astrophysical Observatory)

Kurucz, R. 1994, Atomic data for Fe and Ni, CD-ROM No. 22 (Cambridge, MA: Smithsonian Astrophysical Observatory)

Kurucz, R., \& Bell, B. 1995, CR-ROM No. 23 (Cambridge, Mass.: Smithsonian Astrophysical Observatory)

Kurucz, R. L., \& Peytremann, E. 1975, SAO Spec. Rep., 362

Lanz, T., Artru, M. C., Didelon, P., \& Mathys, G. 1993, A\&A, 272, 465

Lawler, J. E., Wickliffe, M. E., Den Hartog, E. A., \& Sneden, C. 2001a, AJ, 563, 1075

Lawler, J. E., Wickliffe, M. E., Cowley, C. R., \& Sneden, C. 2001b, ApJS, 137, 341

Lawler, J. E., Bonvallet, G., \& Sneden, C. 2001c, AJ, 556, 452

Lawler, J. E., Den Hartog, E. A., Sneden, C., \& Cowan, J. J. 2006, ApJS, 162, 227

Lawler, J. E., Sneden, C., Cowan, J. J., Ivans, I. I., \& Den Hartog, E. A. 2009, ApJS, 182, 51

Ljung, G., Nilsson, H., Asplund, M., \& Johansson, S. 2006, A\&A, 456, 1181

López-García, Z., \& Adelman, S. J. 1994, A\&AS, 107, 353

López-García, Z., \& Adelman, S. J. 1999, A\&A, 137, 227

López-García, Z., Adelman, S. J., \& Pintado, O. I. 2001, A\&A, 367, 859

Makaganiuk, V., Kochukhov, O., Piskunov, N., et al. 2011, A\&A, 529, A160

Martin, G. A., Fuhr, J. R., \& Wiese, W. L. 1988, J. Phys. Chem. Ref. Data, 17, 3

Mathys, G. 1991, A\&AS, 89, 121

Mathys, G. 1995a, A\&A, 293, 733 
Mathys, G. 1995b, A\&A, 293, 746

Mayor, M., Pepe, F., Queloz, D., et al. 2003, The ESO Messenger, 114, 20

Meggers, W. F., Corliss, C. H., \& Scribner, B. F. 1975, Tables of Spectral Line Intensities, 2nd edn. (Washington DC: US Gov. Print. Off.), Mono. 145

Mowlavi, N., Eggenberger, P., Meynet, G., et al. 2012, A\&A, 541, A41

Nielsen, H., Karlsson, H., \& Wahlgren, G. M. 2000, A\&A, 363, 815

Nuñez, N. E., González, J. F., \& Hubrig, S. 2011, in Magnetic Stars, eds. D. O. Kudryavtsev, \& I. I. Romanyuk, 361

Palacios, A., Gebran, M., Josselin, E., et al. 2010, A\&A, 516, A13

Pickering, J. C., Thorne, A. P., \& Perez, R. 2002, ApJS, 138, 247

Press, W. H., \& Rybicki, G. B. 1989, ApJ, 338, 277

Proffitt, C. R., Brage, T., Leckrone, D. S., et al. 1999, ApJ, 512, 942

Reiners, A., \& Schmitt, J. H. M. M. 2002, A\&AS, 384, 155

Renson, P., \& Manfroid, J. 2009, A\&AS, 498, 961

Ryabchikova, T. A., \& Smirnov, Yu. M. 1994, Astron. Rep., 38, 70

Ryabchikova, T., Ryabtsev, A., Kochukhov, O., \& Bagnulo, S. 2006, A\&A, 456, 329
Sbordone, L., Bonifacio, P., Castelli, F., \& Kurucz, R. L. 2004, MSAIS, 5, 93

Schaller, G., Schaerer, D., Meynet, G., \& Maeder, A. 1992, A\&AS, 96, 269 Schöller, M., Correia, S., Hubrig, S., \& Kurtz, D. W. 2012, A\&A, 545, A38 Schulz-Gulde, E. 1969, J. Quant. Spectr. Rad. Transf., 9, 13

Sigut, T. A. A., \& Landstreet, J. D. 1990, MNRAS, 247, 611

Snik, F., Kochukhov, O., Piskunov, N., et al. 2011, ASP Conf. Ser., 437, 237

Warner, B. 1968, MNRAS, 139, 115

Wickliffe, M. E., Lawler, J. E., \& Nave, G. 2000, J. Quant. Spectr. Rad. Trans., 66,363

Wiese, W. L., Smith, M. W., \& Glennon, B. M. 1966, NSRDS-NSB (Washington DC: Us Dept. of Commerce), 4

Wiese, W. L., Fuhr, J. R., \& Deters, T. M. 1996, J. Phys. Chem. Ref. Data, Monograph, 7

Wolnik, S. J., Berthel, R. O., \& Wares, G. W. 1971, ApJ, 166, 31

Woolf, V. M., \& Lambert, D. L. 1999, ApJ, 520, L55

Zavala, R. T., Adelman, S. J., Hummel, C. A., et al. 2007, AJ, 655, 1046 